EPJ Web of Conferences 81, 06008 (2014)

DOI: 10.1051 epjconf/ 20148106008

(C) Owned by the authors, published by EDP Sciences, 2014

\title{
Studies on implementation of pellet tracking in hadron physics experiments
}

\author{
A. Pyszniak ${ }^{1,2, a}$, H. Calén ${ }^{2}$, K. Fransson², V. Hejny ${ }^{3,4}$, T. Johansson², J. Löfgren², Z. Rudy ${ }^{1}$, \\ M. Wolke ${ }^{2}$, and P. Wüstner ${ }^{5,6}$ \\ ${ }^{1}$ Institute of Physics, Jagiellonian University, PL-30-059 Cracow, Poland \\ ${ }^{2}$ Department of Physics and Astronomy, Uppsala University, SE-75120, Uppsala, Sweden \\ ${ }^{3}$ Institut für Kernphysik, Forschungszentrum Jülich, 52425 Jülich, Germany \\ ${ }^{4}$ Jülich Center for Hadron Physics, Forschungszentrum Jülich, 52425 Jülich, Germany \\ ${ }^{5}$ Zentralinstitut für Elektronik, Forschungszentrum Jülich, 52425 Jülich, Germany \\ ${ }^{6}$ Physikalisches Institut, Friedrich-Alexander-Universitaät Erlangen-Nuürnberg, Erwin-Rommel-Str. 1, 91058 \\ Erlangen, Germany
}

\begin{abstract}
A system for optical tracking of frozen hydrogen microsphere targets (pellets) has been designed. It is intended for the upcoming hadron physics experiment PANDA at FAIR, Darmstadt, Germany. With such a tracking system one can reconstruct the positions of the individual pellets at the time of a hadronic interaction in the offline event analysis. This gives information on the position of the primary interaction vertex with an accuracy of a few $100 \mu \mathrm{m}$, which is very useful e.g. for reconstruction of charged particle tracks and secondary vertices and for background suppression. A study has been done at the WASA detector setup (Forschungszentrum Jülich, Germany) to check the possibility of classification of hadronic events as originating in pellets or in background. The study has been done based on the instantaneous rate a Long Range TDC which was used to determine if a pellet was present in the accelerator beam region. It was clearly shown that it is possible to distinguish the two event classes. Also, an experience was gained with operation of two synchronized systems operating in different time scales, as it will also be the case with the optical pellet tracking.
\end{abstract}

\section{Introduction}

Pellets are microscopic spheres of frozen hydrogen (approx. 25 micrometers in diameter), used as a target in hadron physic experiments such as WASA (Forschungszentrum Jülich, Germany) and planned for the future PANDA experiment (GSI, Darmstadt, Germany). Being a discrete target, pellets offer a possibility to measure their positions and times along the way and reconstruct, in the offline event analysis, positions of individual pellets at the time of a hadronic interaction. Such an optical pellet tracking system (PTR) has been designed in Uppsala (ref. [1]) for use at PANDA. The system will give information on the position of the primary interaction vertex with an accuracy of a few $100 \mu \mathrm{m}$. The PTR data will be collected by a standalone system that is synchronized with the main DAQ, since it takes about $100 \mathrm{~ms}$ to collect all measurement data relevant for reconstructing a pellet track and this

a e-mail: andrzej.pyszniak@gmail.com 
time scale is very different from the time scale handled by a hadron physics experiment DAQ (parts of ns to a few $\mu \mathrm{s}$ ). To get experience of how to synchronize and use PTR information in an operating experiment, information from a long-range (LR) TDC at WASA was read out by a standalone system working in parallel to the standard DAQ. This LR TDC system operates with a similar time scale as pellet tracking (between some $\mu$ s and several seconds). The synchronization was achieved by writing a common time stamp (and event number) to both DAQ systems.

This study gives a simple demonstration of one possibility with pellet tracking. Using information from pellet tracking, the number of pellets in the accelerator beam region at the time of an interaction can be reconstructed. This information alone allows suppression, in two ways, of effects due to events not originating from interactions of the accelerator beam with pellets:

1) Event-by-event: reject events occurring when no pellet was present.

2) In measurement distributions: use the no-pellet event sample to correct for background.

This kind of "empty target" correction is normally difficult to make in high luminosity internal target experiments, since the presence of the target strongly influences the beam conditions.

\section{Principle of the study}

There are pellets in the beam only for some fraction of the time, while events due to rest-gas and beam halo happen all the time. When a pellet is in the beam, it is most probable that the event came from the pellet. The rate of interactions is exploited, to know when there are pellets in the beam. When a pellet passes through the beam, there are more interactions. For this purpose one of the trigger signals for collecting elastic scattering events (originating from the nominal interaction point) was connected to the LR TDC and used to monitor accelerator beam - pellet "interactions". A typical $5 \mathrm{~ms}$ time spectrum from the LR TDC is shown in Fig. 1.

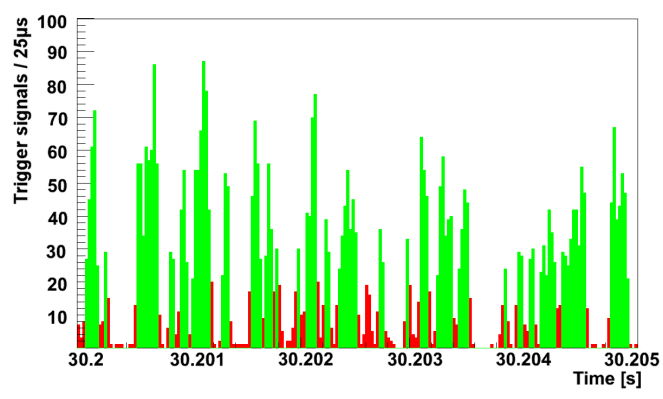

Figure 1. Example TDC spectrum with classification of pellet and non-pellet periods. Green - pellet expected in the beam region, red - no pellet expected in the beam region.

It takes $\approx 70 \mu$ s for a pellet to cross the COSY beam at its center. Structures of such duration are visible in the time spectrum. A simplified classification of time bins, each of length $25 \mu \mathrm{s}$, was done (as indicated in Fig. 1):

- "Non-pellet-class" (red) - low signal rate, 0-20/bin, meaning that there are probably no pellets in the beam region.

- "Pellet-class" (green) - high signal rate, >20/bin, indicating the presence of pellets in the beam region.

The signal rate ranges were adjusted to correct for accelerator beam decay $(\approx 50 \%)$ during the $50 \mathrm{~s}$ period in the accelerator cycle when the beam is present. To check the feasibility of using a standalone (pellet tracking like) system, a 16 min short test run at WASA was performed and the possibility to suppress background events by exploiting the LR TDC information in the event analysis was studied. 
As a test reaction, $p p \rightarrow p p \pi^{0} \rightarrow p p \gamma \gamma$ at a beam kinetic energy of $0.45 \mathrm{GeV}$ was chosen. Events where both protons hit the forward detector and the two gammas hit the very forward part of the electromagnetic calorimeter were specially studied since it is fairly easy to see effects from rest-gas interactions in this kind of events.

\section{Results and discussion}

Fig. 3 shows $\gamma-\gamma$ invariant mass and $p$ - $p$ missing mass distributions for events collected during LR TDC "Non-pellet-class" and "Pellet-class" time intervals. It is clearly visible that the structures originating from background, visible in case of the "Non-pellet-class" are substantially reduced in the "Pellet-class" sample. The results are in good agreement with Monte Carlo simulations for suitable rest-gas contributions. In addition, some structures visible in the "Non-pellet-class" case are probably caused by pile-up events, events originating in the beam pipe walls and other background.

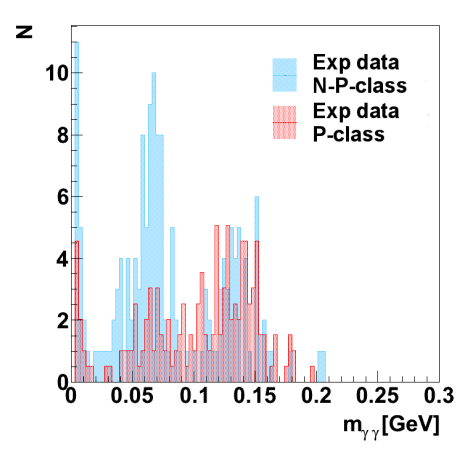

(a) $\gamma-\gamma$ invariant mass

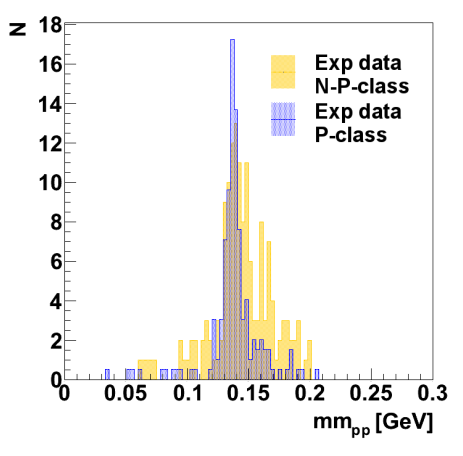

(b) $p$ - $p$ missing mass
Figure 2: Effect of event classification for small azimuthal angles of the gammas $\left(\Theta<40^{\circ}\right)$. "Pellet" and "non-pellet" classes in the experimental data compared with each other.

Detailed vacuum calculations reproducing well all vacuum gauge readings have been done for the WASA setup. They gave pressure levels of the order of 10 times lower than the value required for room temperature rest-gas to cause the measured fraction of background events. The difference might be due to the cryogenic nature of the pellets and the gas conditions close to the pellet stream accelerator beam crossing.

It has been shown that background in the WASA experiment could be reduced by using the knowledge of the number of pellets in the accelerator beam region at a time of a hadronic event.

\section{Acknowledgements}

The project is supported by COSY-FFE, EC FP7, Swedish Research Council, Foundation for Polish Science (MPD program) and Polish National Science Center.

\section{References}

[1] A. Pyszniak et al., Forschungszentrum Jülich IKP Annual Report, (2012), http://www.fzjuelich.de/ikp/EN/Aktuelles/ar2012.html 